\title{
ROTURA DE ANEURISMA DEL SENO DE VALSALVA CORONARIO DERECHO EN PACIENTE FEMENINO: REPORTE DE CASO
}

\section{ANEURYSM OF RIGTH CORONARY SINUS RUPTURE OF VALSALVA IN FEMALE PATIENT: A CASE REPORT}

${ }_{1}$ Profesor Titular en la Unidad de Investigación Científica, FCM-UNAH. MD. PhD.

Cardiólogo Clínico e Intervencionista.

2 Estudiante de la carrera de Medicina, Facultad de Ciencias Médicas Universidad Nacional Autónoma de Honduras (FCMUNAH).

Correspondencia a: Efraín

Antonio Silva Coello correo: efrainsilva.1512@gmail.com

Palabras clave: Seno de Valsalva, Aneurisma, Rotura

Keywords: Valsalva sinus, Aneurysm, Rupture
Procedencia y arbitraje: no comisionado, sometido a arbitraje externo. Recibido para publicación: 21 de abril de 2018 Aceptado para publicación: 04 de junio de 2018

Citar como: Rev Cient Cienc Med 2019; 22(1): 48-52

Gustavo A. Moncada Paz ${ }^{1}$, Efraín Antonio Silva Coello ${ }^{2}$, Julio David San Martin Pavón ${ }^{2}$, Ana Saraí Salgado Fonseca ${ }^{2}$, Obed Josué Morales Mejía ${ }^{2}$.

\section{RESUMEN}

Los aneurismas del seno de Valsalva son anomalías infrecuentes, ocasionadas por la falta de continuidad entre la túnica media de la aorta y el anillo valvular. Suelen ser asintomáticos mientras estén íntegros, la mayoría se diagnostica sucesivamente a una rotura. Son más frecuentes en el sexo masculino y su rotura afecta más a jóvenes adultos. Pueden ser causa de insuficiencia cardiaca y en algunos casos llegan a presentarse como muerte súbita. Su diagnóstico requiere estudios de imagen y su tratamiento generalmente es quirúrgico. Se presenta el caso de un paciente femenino de 18 años que consultó por síntomas de insuficiencia cardiaca. Al examen físico se encontró hipotensa, con choque de punta desplazado hacia la izquierda, soplo auscultado en todos los focos e irradiado hacia la izquierda, ingurgitación yugular y ascitis. La radiografía de tórax reveló cardiomegalia y el ecocardiograma transtorácico evidenció rotura de aneurisma del seno de Valsalva coronario derecho. La sospecha de rotura de aneurisma del seno de Valsalva ante síntomas de insuficiencia cardiaca y soplo, permite la corrección exitosa del defecto.

\section{ABSTRACT}

Aneurysms of the sinus of Valsalva are infrequent anomalies caused by discontinuity between middle tunic of aorta and the valvular ring. Generally, they are asymptomatic while they are intact, most of them are diagnosed after to a rupture. They affect with more frequency males and its rupture mostly occur in young adults; this defect can be a cause of heart failure. In some cases, can occur as sudden death. Its diagnosis requires imaging studies and its treatment is usually surgical. It is presented the case of an 18 years old female patient, who consulted for symptoms of heart failure. Upon physical examination, It was found hipotension with a displaced tip shock, murmur auscultated in all auscultatory sites and it was irradiated to left armpit, presence of jugular ingurgitation and ascites. Chest radiography revealed cardiomegaly and the transthoracic echocardiogram showed rupture of the right coronary sinus of Valsalva aneurysm. Suspect the rupture of the sinus of Valsalva aneurysm at the presence of heart failure symptoms and murmur, allows the successful correction of the defect.

\section{INTRODUCCIÓN}

$\mathrm{E}$ 1 Aneurisma del Seno de Valsalva (ASV) es una anormalidad poco frecuente, afecta a menos del $0,1 \%$ de la población en general, teniendo una prevalencia de $1,2 \%$ a $1,8 \%$ en la población china y de $0,14 \%$ a $0,96 \%$ en la población occidental con cardiopatía congénita ${ }^{1,2}$. La edad de presentación varía ampliamente, presentándose entre los 2 a 74 años con una media de 39 años y predominio en el sexo masculino. Estos aneurismas se pueden clasificar como congénitos o adquiridos, de los cuales los aneurismas congénitos ocurren con mayor frecuencia, siendo responsable del 3,5\% de todas las cardiopatías congénitas, vinculándose además de manera muy frecuente con otros trastornos congénitos como la comunicación interventricular (en su mayoría supracrestal), insuficiencia aórtica $(41,9 \%)$, estenosis pulmonar $(9,7 \%)$, estenosis aórtica $(6,5 \%)$, coartación aórtica $(6,5 \%)$, persistencia del conducto arterioso $(3,2 \%)$ e insuficiencia tricuspidea $(3,2 \%)^{3,4,5}$.

Típicamente este aneurisma se produce en el seno coronario derecho (75-90\%), seguido del seno no coronario (10-25\%) y el resto en el seno coronario izquierdo ${ }^{2}$. El tipo congénito se produce cuando 
existe una falta de fusión entre la capa media de la pared aortica y el anillo fibroso de la válvula aórtica ${ }^{5,6,7}$. El 95\% de estos aneurismas congénitos se originan en el seno coronario derecho o el no coronario. El ASV adquirido puede existir en pacientes con endocarditis, sífilis, lesión, síndrome de Behcet, y el síndrome de Marfan ${ }^{6}$. La complicación más común que presenta este defecto es la rotura, la cual puede ocurrir de manera espontánea, después de un trauma, por ejercicio físico extremo o por endocarditis, aunque esta última también puede ocurrir como una complicación del ASV roto (RASV) ${ }^{8}$.

El RASV ocurre en su mayoría hacía el lado derecho del corazón representando el $60 \%$ de los casos la rotura hacia ventrículo derecho y un $29 \%$ hacia aurícula derecha. Es poco frecuente que ocurra hacia el lado izquierdo o pericardio, representando aproximadamente un $10 \%^{5,9}$. La rotura extracardiaca raramente ocurre, sin embargo, de presentarse es fatal, ocurriendo hacia el espacio pleural y siendo más frecuente en ASV adquiridos'.

A continuación, se presenta el caso de una Rotura de un Aneurisma del Seno de Valsalva Coronario Derecho, siendo el interés de esta publicación registrar la evolución que presenta el paciente tras sufrir esta complicación y ser abordado en las instituciones de salud pública del Honduras de manera que pueda ser tomado en cuenta en casos similares que se pudiesen presentar en un futuro.

\section{PRESENTACIÓN DEL CASO}

Paciente femenino de 18 años, procedente del departamento de Olancho, Honduras, con antecedente en la infancia de soplo infantil, sin causar problemas al paciente durante su infancia y adolescencia. Acudió a un centro asistencial en el departamento de Olancho con historia de disnea de 6 meses de evolución, que progresó de medianos a pequeños esfuerzos, atenuada en reposo, acompañada de ortopnea, disnea paroxística nocturna, palpitaciones, mareos, cianosis generalizada, edema palpebral, edema bilateral de miembros inferiores y ascitis. Se le realizó una ecocardiografía transtorácica que evidenció un aneurisma del seno de Valsalva coronario derecho, razón por la cual fue remitido al "Hospital Escuela Universitario de Tegucigalpa” donde la paciente acudió a dicha institución una semana después.

A su llegada al Hospital, la evaluación física reportó presión arterial de $80 / 40 \mathrm{mmHg}$, frecuencia cardiaca de 97 latidos por minuto, ingurgitación yugular, reflujo hepatoyugular, choque de punta desplazado a sexto espacio intercostal línea axilar anterior, la auscultación cardiaca reveló soplo holosistólico grado III/VI audible en todos los focos de auscultación e irradiado a la axila izquierda y el abdomen se encontró globoso a expensas de ascitis con circulación colateral.

A su ingreso se ordenaron estudios de química sanguínea (Ver tabla 1) y estudios de imagen en los que la radiografía de tórax (Figura 1) mostró un índice cardiotorácico de 0,61 representando una cardiomegalia grado III.

El ecocardiograma transtorácico evidenció rotura de aneurisma del seno de Valsalva coronario derecho con drenaje a aurícula derecha y flujo de izquierda a derecha, dilatación de aurícula y ventrículo derecho, ventrículo izquierdo con fracción de eyección conservada, leve insuficiencia tricuspidea y comunicación interventricular leve (Figura 2).

\begin{tabular}{|c|c|c|}
\hline Examen & Resultado & $\begin{array}{l}\text { Valores de refe- } \\
\text { rencia del hospital }\end{array}$ \\
\hline Glucosa & $56 \mathrm{mg} / \mathrm{dl}$ & $74-106$ \\
\hline BUN & $29 \mathrm{mg} / \mathrm{dl}$ & $7-18$ \\
\hline Sodio & $128 \mathrm{mmol} / \mathrm{L}$ & $136-145$ \\
\hline Calcio & $7,6 \mathrm{mg} / \mathrm{dl}$ & $8,5-10,1$ \\
\hline TGO & $41 \mathrm{U} / \mathrm{L}$ & $15-37$ \\
\hline Ácido Úrico & $8,9 \mathrm{mg} / \mathrm{dl}$ & $2,6-6$ \\
\hline $\begin{array}{l}\text { Bilirrubina } \\
\text { total }\end{array}$ & $1,0 \mathrm{mg} / \mathrm{dl}$ & $0,2-1,0$ \\
\hline $\begin{array}{l}\text { Bilirrubina } \\
\text { directa }\end{array}$ & $0,6 \mathrm{mg} / \mathrm{dl}$ & $0,0-0,2$ \\
\hline $\begin{array}{l}\text { Fosfatasa al- } \\
\text { calina }\end{array}$ & $117 \mathrm{mg} / \mathrm{dl}$ & $46-116$ \\
\hline Albúmina & $2.7 \mathrm{~g} / \mathrm{dl}$ & $3,4-5$ \\
\hline $\begin{array}{l}\text { Creatinina } \\
\text { spital Escuela Unive }\end{array}$ & $\begin{array}{l}1.20 \mathrm{mg} / \mathrm{dl} \\
\text { ario, septiembre de } 2\end{array}$ & $0,5-1,3$ \\
\hline
\end{tabular}

Figura 1: Radiografía PA de Tórax: cardiomegalia grado III.

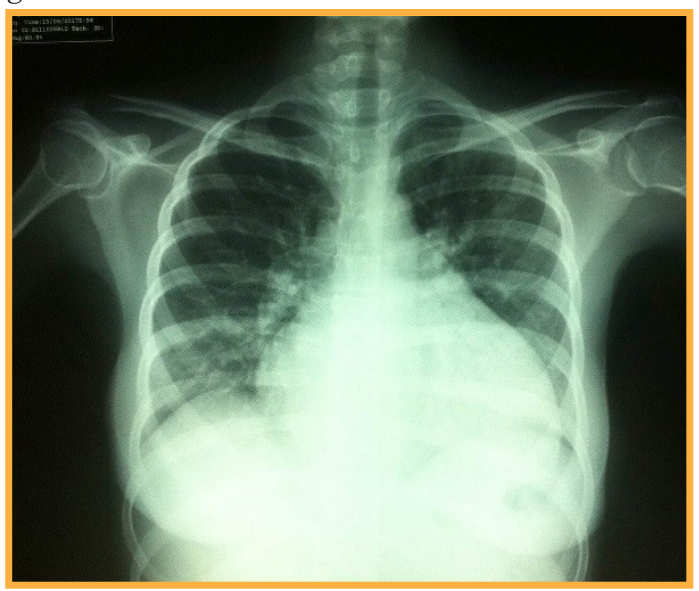

Hospital Escuela Universitario, septiembre 2017 
El electrocardiograma (Figura 3) mostró desviación del eje eléctrico hacia la derecha con inversión de onda $\mathrm{T}$ y ritmo sinusal con trastornos inespecíficos de segmentos y ondas (ST-T) en las derivaciones V1 a V6.

Todos los RASV deben ser considerados como diagnóstico diferencial de pacientes que muestran síntomas de insuficiencia cardíaca aguda con dolor en el pecho y nuevos soplos cardíacos ${ }^{10}$. Teniendo en cuenta la aparición de este último elemento clínico en el curso de dicha complicación, el diagnóstico diferencial puede girar en torno a él, siendo las causas identificadas de soplo continuo: persistencia de conducto arterioso, ventana aortopulmonar, fístulas arteriovenosas coronarias, tronco arterioso, zumbido o Hum venoso soplo mamario gestacional ${ }^{3}$.

El tratamiento quirúrgico se basó en plastia del seno

Figura 2: Radiografía PA de Tórax: cardiomegalia grado III

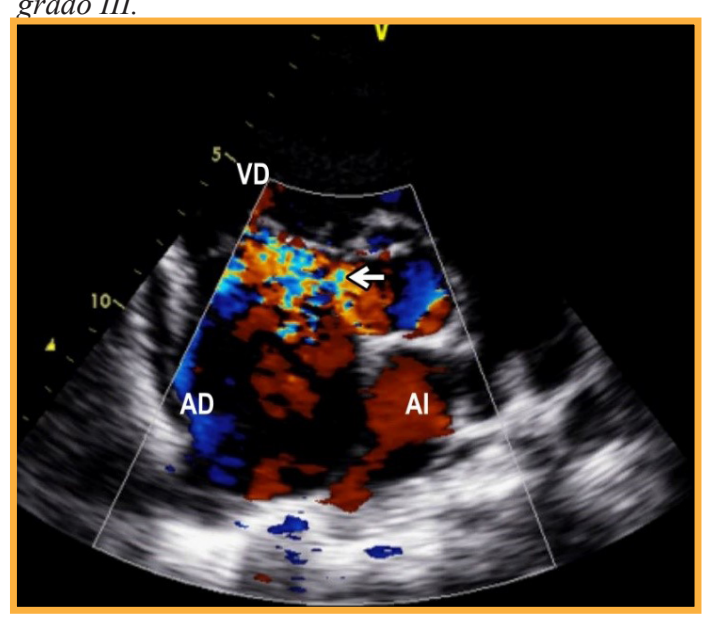

Centro Médico Monte Sinai, Catacamas, Olancho, septiembre 2017. Imagen: Dra: Sherrie Lynch y Dr. Allan Peralta de Valsava bajo circulación extracorpórea en la cual se reparó la rotura de dicho seno de aproximadamente $0,5 \mathrm{~cm}$ con comunicación izquierda-derecha, no se encontró comunicación interventricular y no se presentaron complicaciones durante ni después de la cirugía. El paciente fue dado de alta seis días después de la cirugía sin presentar complicaciones en su seguimiento.

Se recomienda el control riguroso de la presión arterial para todos los pacientes con reparación quirúrgica, cuando el diámetro de la raíz aórtica supera los $5 \mathrm{~cm}$. La asociación con insuficiencia aórtica puede exigir la sustitución de la válvula aórtica, además de la reparación del aneurisma ${ }^{2}$. Las complicaciones graves de la rotura, combinada con las bajas tasas de mortalidad perioperatoria, han dado lugar a que algunos autores recomienden intervención quirúrgica en pacientes asintomáticos ${ }^{2,9}$. EL protocolo de tratamiento principal de un RASV es la cirugía, mientras que la oclusión transcatéter es también aplicable para los pacientes con contraindicaciones quirúrgicas o inestabilidad hemodinámica.

\section{DISCUSIÓN}

Los pacientes con ASV pueden permanecer asintomáticos durante varios años, incluso si el ASV se ha roto. Sin embargo, la disnea y la intolerancia al ejercicio pueden desarrollarse debido al aumento de la derivación y el volumen de sobrecarga ${ }^{6}$.

Un ASV puede agrandarse hasta ocasionar complicaciones como compresión de la cámara adyacente, compresión de la arteria coronaria, bloqueo cardiaco completo y muerte súbita cardiaca. Sin embargo, solo en pocos pacientes se

Figura 3: Electrocardiograma: ritmo sinusal con trastornos inespecificos de segmentos y ondas (ST-T) en V1 a V6.

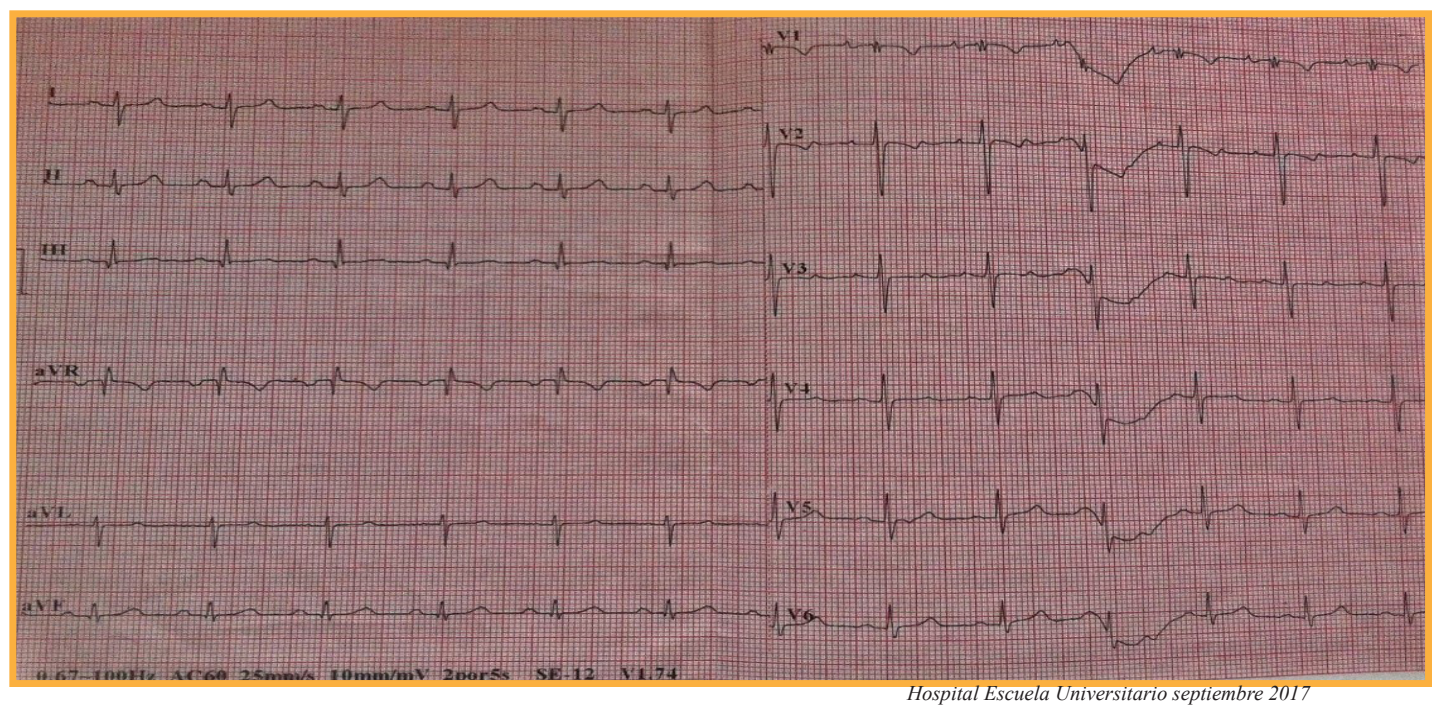


observa obstrucción mecánica, regurgitación y otros cambios anatómicos y fisiológicos que puedan producir síntomas destacados como arritmias, tromboembolismo o isquemia cardiaca ${ }^{11,12}$.

Una vez que el aneurisma se rompe en la aurícula, ventrículo o cavidad pericárdica, puede aparecer insuficiencia cardíaca aguda o hemorragia catastrófica. La presentación de los síntomas es progresiva, siendo los más frecuentes: fatiga, disnea y dolor torácico; sin embargo, los pacientes con RASV pueden ser asintomáticos y presentar solamente un soplo durante la auscultación. En el caso del paciente, presentó síntomas de insuficiencia cardiaca como disnea progresiva aliviada en reposo, ortopnea, disnea paroxística nocturna y palpitaciones ${ }^{9}$.

En la exploración física las personas con RASV comúnmente presentan un soplo continuo a la auscultación mesocárdica, no obstante, en determinadas ocasiones se puede auscultar solo un soplo sistólico o una combinación de un soplo sistólico con un soplo diastólico. En este caso se encontró soplo holosistólico grado III/VI audible en todos los focos de auscultación irradiado a la axila izquierda.

Los hallazgos electrocardiográficos en aneurisma del seno de Valsalva roto son hipertrofia ventricular, eje eléctrico hacia la derecha y fibrilación auricular. En aneurismas del seno de Valsalva no rotos por lo general el electrocardiograma es normal, a menos que el aneurisma comprima el nodo o sus ramas atrioventriculares, generando bloqueo completo $\mathrm{u}$ otras alteraciones de la conducción auriculoventricular ${ }^{9}$. En este caso el paciente presentó eje eléctrico desviado hacia la derecha y ritmo sinusal con trastornos inespecíficos de segmentos y ondas (ST-T), no presentó fibrilación auricular.

La ecocardiografía transtorácica y la ecocardiografía transesofágica representan los principales métodos de diagnóstico ${ }^{10,11,13}$. La ecocardiografía transtorácica bidimensional puede detectar hasta el $75 \%$ de todos los pacientes con aneurisma de seno de Valsalva. La cateterización cardiaca con aortografía también es considerada al igual que la ecocardiografía como estándar de oro para el diagnóstico ${ }^{2,13}$. Sin embargo, las técnicas de imagen alternativas como resonancia magnética, tomografía computarizada y resonancia magnética nuclear se han propuesto anteriormente para el diagnóstico de aneurismas del seno de Valsalva ${ }^{2,10,11}$. En este caso se utilizó para el diagnóstico la ecocardiografía transtorácica.

Las técnicas recomendadas para cerrar la RASV incluye el cierre con parche o reparación directa utilizando suturas interrumpidas. El cierre primario de la RASV tiene desventajas potenciales como la deformación del seno aórtico; la interrupción de la competencia aórtica y el estrés de la línea de sutura conducen a la recurrencia. Dicha recurrencia y el empeoramiento de la aurícula derecha han sido reportados después de un cierre primario. El cierre con parche se recomienda en la RASV $(60,3 \% \pm$ $26,4 \%$ ) en los casos de reportes revisados ya que evita la deformidad de la válvula aórtica y reduce la presión sobre la línea de sutura ${ }^{14} \mathrm{El}$ dispositivo de cierre percutáneo de la RASV es una alternativa para traumas menores desde que Cullen reportó el primer caso de cierre transcatéter de la Ruptura del Seno de Valsalva ${ }^{6}$. En este caso se realizó plastía del aneurisma del seno de Valsalva bajo circulación extracorpórea.

La mortalidad operatoria de la reparación de la RASV es 0,5 a $61 \%$ con un buen pronóstico a largo plazo14. En los pacientes que presentan RASV no intervenido, se ha reportado una supervivencia media de 3,9 años. Estos reportes apoyan la necesidad de una intervención quirúrgica temprana. Por otro lado, los resultados quirúrgicos de la reparación de la RASV muestran una supervivencia mayor del $90 \%$ a los veinte años ${ }^{9}$.

\section{CONClusión}

Aunque no ocurra muy a menudo, los pacientes con síntomas de insuficiencia cardiaca acompañados de soplos deben despertar la sospecha de la rotura de un Aneurisma del Seno de Valsava para considerar un manejo adecuado y evitar complicaciones que podrían ser fatales para el paciente.

\section{Agradecimientos}

Agradecemos a la Dra. Sherrie Lynch y al Dr. Allan Peralta del Centro Médico Monte Sinaí de la ciudad de Catacamas por su valiosa colaboración, sin la cual no hubiese sido posible realizar este reporte de caso.

Aneurisma del seno de valsava derecho roto 
1. Yang Y, Zhang L, Wang X, Lü Q, He L, Wang J, et al. Echocardiographic diagnosis of rare pathological patterns of sinus of Valsalva aneurysm. PLoS ONE [internet]. 2017;12(3):e0173122. Acceso 23 de Septiembre de 2017. Disponible en: https://www. ncbi.nlm.nih.gov/pmc/articles/PMC5349664/pdf/ pone.0173122.pdf.

2. Bamford P, Collins N. Severe Dilatation of Coronary Artery Ostium Complicating Sinus of Valsalva Aneurysm: Differential Diagnosis and Review of the Literature. Case Rep Cardiol [internet]. 2017;8694652. Acceso 23 de Septiembre de 2017. Disponible en: https://www.ncbi.nlm.nih.gov/pmc/ articles/PMC5406726/pdf/CRIC2017-8694652.pdf.

3.Tápanes Daumy $H$, Peña Fernández $M$, Savío Benavides A. Soplo continuo: un signo de inestimable valor. Sociedad Cubana de Cardiología [internet]. 2013;5(3):285-95. Acceso 24 de Septiembre de 2017. Disponible en: $h t t p: / / b v s . s l d . c u /$ revistas/cors/pdf/2013/ v5n3a13/es/corsalud5(3)2013.pdf

4. McGregor P-C, Temtanakitpaisan Y, Hiltbolt A, Aragam JR.A spectrum of sinus of Valsalva aneurysmFrom the young to the old. Echocardiography [internet]. 2017;34(10):1524-30. Acceso 23 de Septiembre de 2017. Disponible en: https://onlinelibrary. wiley.com/doi/pdf/10.1111/echo.13655

5. Godinez-Baca L, Luna-Pérez D. Ruptura de seno de Valsalva como causa de insuficiencia cardiaca aguda en un paciente joven. Rev Mex Cardiol [Internet]. 2016;27(1):52-5. Acceso 23 de septiembre de 2017. Disponible en: http://www.medigraphic.com/pdfs/ cardio/h-2016/hs161g.pdf.

6. Kumar GA, Parimala PS, Jayaranganath M, Jagadeesh AM. Three-dimensional transesophageal echocardiography-guided transcathetar closure of ruptured noncoronary sinus of valsalva aneurysm. Ann Card Anaesth [internet]. 2017;20(1):73-75. Acceso 23 de Septiembre de 2017. Disponible en: $h t t p: / / w w w$. annals.in/article.asp?issn $=0971-9784$;year $=2017$; volume $=20$; issue $=5$; page $=73$; page $=75$; aulast $=$ Kumar

7. Hernández ADM, Jiménez DP. Ruptura de aneurisma de Valsalva no coronario. Medisur [Internet]. 2016;14(4):495-8. Acceso 23 de Septiembre de 2017. Disponible en: $h t t p: / / m e d i s u r . s l d . c u / i n d e x . p h p /$ medisur/article/download/3277/2068.
8. Davarpasand T, Hosseinsabet A, Abbasi K. Ruptured Sinus of Valsalva Aneurysm Accompanied by Aortic and Tricuspid Valve Endocarditis. The Journal of Tehran University Heart Center [Internet]. 2017;12(1):42-5. Acceso 24 de Septiembre de 2017. Disponible en: https://www.ncbi.nlm.nih.gov/pmc/ articles/PMC5409950/

9. González N, Schlack E, Flores P, Astudillo C. Aneurisma del seno de valsalva: Presentación de tres casos. Revista chilena de cardiología [internet]. 2016;35(3):249-54. Acceso 23 de Septiembre de 2017. Disponible en: https://scielo.conicyt.cl/pdf/rchcardiol/ v35n3/art06.pdf

10. Zhu E, Westenfeld R, Gastl M, Bönner F, Assmann A, Nia AM, et al. Acute chest pain in a triathlete: Rupture of the noncoronary sinus of Valsalva into the right ventricle. J Thorac Dis [internet]. 2016;8(10):1199-1201. Acceso 23 de Septiembre de 2017. Disponible en: https://www.ncbi.nlm.nih.gov/pmc/ articles/PMC5107541/

11. Du R, Liang Z, Li X, Ji Y, Zhong T, Deng Z. Ruptured noncoronary sinus of Valsalva aneurysm into the right atrium: a rare cause of pulmonary thrombus with pneumonia. Int J Clin Exp Pathol [internet]. 2017;10(5):5994-8. Acceso 24 de Septiembre de 2017. Disponible en: http://www.ijcep.com/files/ijcep0043438. $p d f$

12. Patel N, Rousan TA, Peyton MD, Sivaram CA. Two different presentations of sinus of valsalva aneurysm. Echocardiography [internet]. 2014;31(6):181-4. Acceso 23 de Septiembre de 2017. Disponible en: https:// onlinelibrary.wiley.com/doi/abs/10.1111/echo.12593

13. Rajan R, Amin O, Soman B, Dashti R, Al Jarallah $M$. Rupture of noncoronary sinus of Valsalva into right atrium in the fifth decade of life. Heart India [internet]. 2017;5:125-6. Acceso 25 de Septiembre de 2017. Disponible en: http://www.heartindia.net/text. asp?2017/5/3/125/214424

14. Gupta M, Shoeb M, Mishra PK, Bathla A, Prasad $\mathrm{J}$. Management of the ruptured sinus of valsalva aneurysmsa. Int Surg J [internet]. 2017;4(6):1873. Acceso 20 de Septiembre de 2017. Disponible en: $h t t p: / /$ www.ijsurgery.com/index.php/isj/article/view/1348 